\title{
Reflexiones sobre Armenia hoy Una visita a Ereván
}

\author{
Ricardo Torres ${ }^{*}$
}

En la segunda mitad de septiembre de 2017, volví a Ereván, capital de Armenia, donde había estado en 2003 para realizar entrevistas para mi tesis doctoral sobre el conflicto de Nagorno-Karabagh -que los armenios mantienen con Azerbaiyán- y sus consecuencias sobre el nacionalismo armenio (1998-2016).

Mi primera impresión al llegar a Ereván fue favorable ya que el aeropuerto internacional de Zvartnots, $12 \mathrm{Km}$ al oeste de la ciudad, es una construcción moderna muy diferente del viejo aeropuerto soviético que yo había conocido. Cabe destacar que el mismo está gerenciado en la actualidad por Armenia International Airports de Eduardo Eurnekian, responsable de las obras de ampliación y mejoras a partir de 2004.

Vale la pena en este momento hacer una breve introducción sobre Ereván, colocándola en la geografía y en la historia de Armenia. Ereván es la capital de Armenia desde 1918 (de la Primera República en 1918-1920, de la República Socialista Soviética en 1920-1991 y de la Tercera República desde 1991) ${ }^{1}$. La ciudad está ubicada en las márgenes del río Hrazdan, en las llanuras de Ararat, en el altiplano armenio $^{2}$ y a sólo $23 \mathrm{~km}$ de la frontera entre Armenia y Turquía cerrada en 1993. Con una altura promedio entre los 900 y los 1.400 msnm, Ereván está rodeada en su parte alta por montañas en tres lados, mientras que en su parte inferior, el río Hrazdan forma un cañón que divide a la ciudad en dos partes. El monte Ararat, volcán apagado formado por dos picos: el Gran Ararat de $5.137 \mathrm{~m}$ y el Pequeño Ararat (Monte Sis) de $3.896 \mathrm{~m}$, actualmente en Turquía y a $32 \mathrm{~km}$ de la frontera armeno-turca, hacia el sur de Ereván, se puede distinguir perfectamente desde todo Ereván. Ararat, considerado el lugar mítico donde se posó el arca de Noé, es un símbolo nacional armenio y está representado en el escudo de armas del país. Los volcanes extinguidos de Aragats de 4.090 m y Azhdahak de 3.597 m están al norte y al este de la ciudad.

Antes de entrar en la historia y arquitectura de Ereván, y con ellas de algún modo en la de Armenia, es importante destacar tres temas de vital importancia que marcaron la historia

\footnotetext{
* Doctorando en Relaciones Internacionales, Universidad Nacional de Rosario (UNR, Argentina); MBA (Dartmouth College); MIM (Thunderbird); Licenciado en Economía y Administración de Empresas, Universidad Católica Argentina. Trabajó para Reuters Ltd. entre 1986 y 2003 siendo Director General para Rusia y el CEI entre 2001 y 2003. Correo electrónico: rtorres1957@ hotmail.com

${ }_{1}^{1}$ Ereván es la doceava capital histórica de Armenia como Estado independiente desde el 331 a.C. hasta la fecha. Seis de las capitales históricas (Yervandashat, Tigranakert, Bagaran, Shirakavan, Kars y Ani) estaban ubicadas en Armenia Occidental y una (Sis) en Cilicia, ambas regiones actualmente en Turquía. Sólo cinco se encuentran en el actual territorio de la República de Armenia (Armavir, Artashat, Vagharshapat, Dvin y Ereván), llamado históricamente Armenia Oriental. El territorio de la actual República de Armenia de $29.743 \mathrm{~km}^{2}$ representa aproximadamente un 8-9 \% del territorio del reino de Armenia en el 301 d.C., bajo la dinastía de los Arsacid o Arshakuni (331 a.C. al 428 d.C.), y un $20 \%$ del territorio del reino de Armenia en el año 1000, bajo la dinastía de los Bagratuni (884-1045). La mayor parte de las tierras históricas de Armenia están ocupadas por Turquía en la actualidad.

${ }^{2}$ El altiplano armenio es una meseta con una superficie aproximada de $400.000 \mathrm{~km}^{2}$ y está ubicado entre el altiplano de Anatolia y el altiplano iraní y limita hacia el norte con las Montañas del Cáucaso y hacia el sur con la Mesopotamia.
} 
presente de ese pueblo: el surgimiento del nacionalismo a fines del siglo XIX, el genocidio a manos de los otomanos en 1915-1923 y la guerra de Nagorno-Karabagh.

Con respecto al nacionalismo cabe destacar el aporte de la literatura que, a mediados del siglo XIX, contribuyó a generar un renacimiento intelectual que no se había visto en Armenia desde el siglo V d.C. El romanticismo fue la respuesta que encontraron los escritores para construir un nuevo nacionalismo que a su vez llevaría a la insurrección, particularmente entre los armenios orientales (Oshagan, 2004:164-5; Panossian, 2006:142).

Consecuentemente, el fin del siglo XIX vio surgir también los primeros partidos revolucionarios armenios con distintas actitudes sobre la situación de los armenios, particularmente en Armenia Occidental (Imperio Otomano). El Partido Revolucionario Hnchakian, luego llamado el Partido Hnchakian Social Demócrata, fue el primer partido político armenio con una base nacional e internacional y un programa político claro. Fundado en Ginebra, en 1887, por Maro Vardanian, Avetis Nazarbekian, y otros intelectuales armenios orientales que habían sido influidos por el marxismo y el populismo ruso, el partido tomó el nombre de su periódico Hnchak (Campana) que se había inspirado en el periódico ruso Kolokol (Campana) de Alexander Herzen ${ }^{3}$ (Hovannisian, 2004:214-215).

Mientras tanto, en la Armenia Oriental, las medidas represivas de Alejandro III fomentaron la agitación revolucionaria, particularmente de aquellos intelectuales armenios que habían pasado por las sociedades secretas rusas Zemlia $i$ Volia (Tierra y Voluntad) y Narodnia Volia (Voluntad Popular). Tiflis, la ciudad con la mayor comunidad armenia, se convirtió en la sede de numerosas organizaciones legales, semi legales e ilegales armenias. Las más revolucionarias intentaron estructurar organizaciones guerrilleras para ser infiltradas en Armenia Occidental, llamada erkir (patria) por los armenios (Hovannisian, 2004:215).

En 1890 se fundó en Tiflis la Federación de los Revolucionarios Armenios (Hai Heghapokhakanneri Dashnaksutiun). Si bien sus miembros fundadores - Kritapor Mikayelian, Simon Zavarian y Stepan Zorian (Rostom)- tenían orientación socialista, evitaron ser identificados como tales con el objetivo de formar una coalición lo más amplia posible. Sin embargo, lograron convencer a sus seguidores de que la libertad política y económica de Armenia estaba basada en los principios del socialismo (Hovannisian, 2004:215-6).

$\mathrm{Si}$ bien no es posible determinar con exactitud cuan prevalentes eran las ideas nacionalistas entre la población armenia en general, se podría sostener que tanto la identidad nacional como el nacionalismo tenían una amplia base de apoyo en la última década del siglo XIX (Panossian, 2006:176). A fines del siglo XIX, había surgido una identidad nacional pan armenia gracias a que la ideología política prevalecía como fuerza unificadora sobre las diferencias culturales y lingüísticas entre armenios orientales y occidentales y a la supremacía del radicalismo nacionalista de los armenios orientales en el ámbito pan armenio. La nación armenia tenía sus idiomas vernáculos, literatura, liderazgo político y organizaciones pan nacionales, aunque los armenios no fueran la mayoría en sus territorios históricos, salvo en algunos distritos. La reconstrucción de la identidad armenia en el siglo XIX se basó sobre principios nacionales antes que religiosos (Panossian, 2006:178-9).

Luego del renacer del nacionalismo al final del período soviético, también hubo cambios en la posición de la diáspora con respecto a Armenia. Nagorno-Karabagh y la

\footnotetext{
${ }^{3}$ El objetivo de los Hnachakistas era la emancipación de Armenia Occidental (otomana) para el corto plazo y la creación de un Estado socialista independiente dentro de un orden socialista internacional, a largo plazo. Altamente centralizados, los órganos centrales del partido permanecieron en Ginebra mientras extendían sus actividades en el Imperio Otomano y Rusia (Hovannisian, 2004:214-215).

${ }^{4}$ Cuando se realizó el primer congreso en 1892, la organización había cambiado su nombre por el de Federación Revolucionaria Armenia (Hai Heghapikhakan Dashnaktsutiun) y aprobado un programa político. Las diferencias fundamentales entre los dos partidos se centraron en que los Dashnaks buscaban la libertad y autonomía de Armenia dentro del Imperio Otomano, mientras que los Hnachakistas buscaban la separación e independencia de Armenia Occidental (otomana) (Hoannisian, 2004:215-6).
} 
independencia de Armenia en 1991 llevaron de hecho a modificar la idealización de la patria conceptualizada en la Causa Armenia o "Hay Tad" (Libaridian, 1999:82) al constatar la realidad de una lucha de liberación nacional en Nagorno-Karabagh y una Armenia independiente que necesitaba el apoyo de la diáspora. Se pasó de la temporalización de la Armenia Soviética como un museo y una patria temporaria hasta la reunificación con las tierras históricas de Armenia Occidental (Libaridian, 1999:124) a que fueran consideradas como el verdadero foco del pensamiento de la diáspora en referencia a la patria de los armenios. Se llegó al reconocimiento de una realidad sin abandonar el reclamo territorial que adquiere particular relevancia en virtud del centenario del genocidio en 2015.

Con respecto al Genocidio perpetrado por los otomanos entre 1915-1923, la cifra generalmente aceptada de las víctimas armenias es de 1.500.000, aunque hay variaciones según los autores que en esa cifra incluyen asesinatos y deportaciones (Walker, 2004:273-4).

El genocidio, que no ha sido reconocido por Turquía, ha marcado la identidad nacional armenia, particularmente en la diáspora. De este modo, la causa armenia se ha territorializado, ya que muchos demandan el retorno de los territorios perdidos en Armenia Occidental.

La guerra de Nagorno-Karabagh surgió en 1988, al final del período soviético, cuando comenzaron las manifestaciones en Armenia y en Nagorno-Karabagh en pro de la unificación de Nagorno-Karabagh -territorio de mayoría armenia que formaba una región autónoma bajo control de Azerbaiyán- con Armenia. Estos movimientos fueron enfrentados con pogroms en Sumgait, Azerbaiyan, donde docenas de armenios murieron, seguidos por otras manifestaciones violentas en Bakú y Kirovabad (Ganja) en los dos años siguientes. Cerca de 400.000 armenios dejaron Azerbaiyán y 170.000 azeríes dejaron Armenia en medio de tensiones nacionalistas. Mientras tanto, Armenia y Nagorno-Karabagh invocaron la ley soviética pidiendo un cambio administrativo. A fines de 1990 y comienzos de 1991, el ejército soviético participó en acciones militares apoyado por unidades azeríes, las cuales forzaron el éxodo de entre 150.000 y 200.000 armenios que habitaban el norte de Nagorno-Karabagh. En la primavera de 1991 el conflicto comenzó a militarizarse. Luego del fracasado golpe en Moscú de agosto de 1991, Azerbaiyán proclamó su independencia. En septiembre de ese año Nagorno-Karabagh informó que no deseaba seguir formando parte de Azerbaiyán, y proclamó su propia independencia, ratificada ese mismo diciembre. Las acciones militares se intensificaron, primero con una ofensiva de Azerbaiyán desde el sur, entre diciembre 1991 y mayo de 1992, interrumpida por las victorias armenias en Khojali, Shushi (Shusha en azerí) y Lachin. A esto le siguió una segunda fase en el verano de 1992 con una fuerte ofensiva de Azerbaiyán apoyada por mercenarios extranjeros que ocuparon la mitad de Nagorno-Karabagh. Los armenios contra atacaron a partir de octubre de 1992 y hasta septiembre de 1993 cuando la mayor parte de Nagorno-Karabagh fue liberado y se ocuparon $5.500 \mathrm{~km}^{2}$ de territorio de Azerbaiyán. Desde Bakú se intentó una contra ofensiva en septiembre de 1993 sin mayores resultados. Cuando se firmó el armisticio en mayo de 1994, las fuerzas armenias ocupaban casi todo Nagorno-Karabagh y siete distritos de Arzerbaiyán, situación que se mantiene hasta la actualidad. Los incidentes en la línea de separación han sido constantes desde entonces, sin embargo no se ha desplegado una fuerza de paz. Las conversaciones que comenzaron en los 90 no han dado resultado hasta el presente (Chaliand, 1994:XIV-XV; Croissant, 1998:41; Hakobyan, 2010:90-95).

\section{Ereván: su historia}

Ereván fue fundada en el 783-782 a. C. en la fortaleza de Erebuni -que aún se puede visitar- por órdenes del rey Argishti de Urartu, por lo que está considerada una de las ciudades más antiguas del mundo continuamente habitadas (Russell, 2004:27). Ereván cayó en manos de los medos y luego de los iraníes. Bajo la dinastía de los Aqueménidas, Erebuni fue uno de los principales centros de la satrapía (provincia) de Armenia. Erebuni adquirió bajo dominio aqueménida el carácter de una ciudad iraní. Al establecerse el primer reino de Armenia bajo las sucesivas dinastías de los Orontid, los Artaxiad y los Arsacid entre el 331 a.C. y el 428 d.C., 
Erebuni perdió importancia estratégica, ya que las primeras dinastías armenias desarrollaron otras ciudades y fundaron otras capitales.

La situación no cambió durante las sucesivas ocupaciones de los iraníes sasánidas entre el 428 d.C y 658 d.C., de los árabes entre el 658 d.C. ${ }^{5}$ y mediados del siglo VIII, de los Bagratuni desde mediados del siglo VIII hasta 1045, de los bizantinos desde 1045 hasta 1064, de los turcos selyúcidas entre 1064 y el siglo XIII, de los mongoles durante los siglos XIII y XIV ya sea directamente o a través de sus vasallos los Zakarid, y de las tribus turcas de los Kara Koyonlu y AqKoyonlu entre los siglos XIV y XVI.

La ciudad sufrió, no obstante, las devastaciones de la invasión de Tamerlán entre 13871402 y del líder turco Qara Iskander entre 1420 y 1436. El retorno del Catholicos (líder espiritual) de la Iglesia Apostólica Armenia a Vagharshapat, en las afueras de Ereván, desde Sis en Cilicia, en 1441, le devolvió a la ciudad una centralidad importante para los armenios (Kouymjian, 2004: 1-7).

A comienzos del siglo XVI, toda la Armenia Oriental incluida Ereván quedó bajo dominio de los safávidas de Irán y de las dinastías que le sucedieron a partir de 1736, que la controlaron hasta 1828 , salvo breves períodos bajo control otomano ${ }^{6}(1582-1604,1635-1636$ y 1724-1736). Durante el reinado de Abbas I de Irán a fines del siglo XVI y comienzos del siglo XVII, muchos armenios fueron deportados a Irán y los musulmanes (iraníes, turcos, kurdos y azeríes) pasaron a representar gradualmente el $70 \%$ de la población para comienzos del siglo XIX. En 1827 los rusos ocuparon Ereván durante la guerra ruso-iraní de 1826-1828 y vieron confirmada su posesión de la ciudad y de Armenia Oriental por el Tratado de Turkmenchay de 1828. Ereván tenía en 1830 aproximadamente 11.400 habitantes, de los cuales había 7.000 musulmanes y 4.000 armenios (Kouymjian, 2004:14-21; Bournoutian, 2004:81, 106; Suny, 2004:109-112).

Los rusos favorecieron una política de resettlement de armenios de Irán y del Imperio Otomano en la ciudad. Ereván fue escogida como capital de la Región (Oblast) Armenia hasta 1840, se incorporó a la Gobernación (Gubernia) de Georgia-Imeretia en 1840 y pasó a ser la capital de la nueva Gobernación (Gubernia) de Ereván en 1849, situación que se mantuvo hasta la caída del Imperio Ruso en 1917. En 1836, el Estatuto (Polozhenie) reguló las relaciones entre el gobierno zarista y la Iglesia Apostólica Armenia, que recibió una autonomía considerable. También la sede de Echmiadzin en Vagharshapat recibió la primacía sobre las diócesis armenias en Rusia. La ciudad creció considerablemente bajo el domino ruso, pero mantuvo el carácter de una ciudad provincial iraní, a pesar de las nuevas construcciones europeas. Se abrieron nuevas escuelas y se construyeron nuevas iglesias. La primera imprenta se instaló en la ciudad y Ereván contó con un teatro europeo por primera vez. La calle Astafyan, centro de la vida comercial europea de la ciudad fue inaugurada, instalándose en la ciudad las primeras fábricas de cognac y bebidas alcohólicas. En 1879, Alejandro II de Rusia concedió a Ereván el estatus de ciudad. A comienzos del siglo XX, Ereván tenía entre 29.000 y 30.000 habitantes. A comienzos del siglo XX, el ferrocarril unió Ereván con Alexandropol (Gyumri), Tiflis (Tbilisi) y Julfa, y la primera biblioteca pública de la ciudad abrió sus puertas (Suny, 2004:113-126).

La guerra entre armenios y azeríes en el Cáucaso en Rusia, entre 1905y 1907, reforzó el carácter nacional de los Dashnaks ya que los llevó a proteger los intereses de la Iglesia Apostólica y de las clases altas de la sociedad armenia en el Cáucaso ruso a la que antes habían criticado, ante los ataques del régimen zarista (Dasnabedian, 2013:95, 105).

Luego de la revolución de octubre de 1917 (noviembre según el calendario gregoriano) y la toma del poder por los bolcheviques en Petrogrado, los líderes georgianos, armenios y musulmanes (azeríes) del Cáucaso del Sur formaron la Federación Transcaucásica, de corta vida, pues se disolvió ante el avance de las fuerzas otomanas y el retiro ruso en las postrimerías de la Primera Guerra Mundial. Luego del Tratado de Batum, el 28 de mayo de 1918, en Tiflis

\footnotetext{
${ }^{5}$ A partir del siglo VII, Erebuni comienzó a ser llamada Ereván.

${ }^{6}$ Los otomanos llamaron Revan a Ereván.
} 
(Tbilisi), la capital de Georgia, el Consejo Nacional Armenio proclamó la Primera República de Armenia con capital en Ereván, en circunstancias humillantes para los armenios. Los Dashnaks controlaron la Primera República hasta su caída en 1920. Cerca de 75.000 refugiados de Armenia Occidental que lograron escapar del genocidio perpetrado por el Imperio Otomano llegaron a Ereván en 1919. Mientras el Tratado de Sèvres abría la puerta a la creación de una Gran Armenia en 1920, ante el avance turco, los bolcheviques ocuparon el país el 4 de diciembre de 1920 proclamando la República Socialista Soviética (Hovannisian, 2004: 284-301; 330-346).

Bajo el régimen soviético, y a pesar de las numerosas restricciones a las libertades individuales y a la represión de cualquier intento nacionalista, Armenia se benefició de su integración en la economía soviética y alcanzó un alto grado de industrialización. Las olas importantes de inmigración armenia entre 1921 y 1936 y de 1946 a 1948 acentuaron el perfil monoétnico de Armenia, extendiéndose el uso del idioma armenio. Armenia sufrió muchas pérdidas humanas durante la Segunda Guerra especialmente como porcentaje de su población. En los años sesenta comenzaron los primeros reclamos nacionalistas (Suny, 2004:347-387).

El Terremoto de 1988, el derrumbe del régimen soviético, la independencia en 1991 y la guerra de Nagorno-Karabagh, junto a las penurias de los años 90, trajeron nuevos desafíos a Armenia y a Ereván. La ciudad y el país tuvieron que adaptarse a una economía en guerra -con las fronteras cerradas con dos de sus cuatro vecinos (Turquía y Azerbaiyán), situación que continúa hasta la actualidad- y a una alta tasa de emigración. Sólo en este siglo, y debido en gran parte a la ayuda de la diáspora, la economía comenzó a mostrar signos de revitalización.

\section{Ereván: su arquitectura}

Bajo el régimen soviético se aprobó un plan urbanístico integral diseñado por el arquitecto Alexander Tamanian en 1924.Tamanian incorporó las tradiciones urbanísticas nacionales en su plan, radial circular, que incorporó muchas de las calles existentes. Muchos edificios históricos (iglesias, mezquitas, la fortaleza iraní, baños públicos, bazares y caravanserais) fueron demolidos. Numerosos distritos de Ereván que rodeaban la zona central (Arabkir, Malatia-Sebastia y Nork-Marash) fueron bautizados con el nombre de poblaciones en Armenia Occidental destruidas por los otomanos durante el genocidio.

Ereván sufrió una disminución de su población ya que pasó de tener 1.2 millones de habitantes en 1989 -bajo el régimen soviético- a tener un poco más de un millón en 2011, debido fundamentalmente a la emigración de los armenios en busca de mejores condiciones de vida. La minoría azerí dejó Ereván a fines de los años 80, al comenzar el conflicto de NagornoKarabagh, y la mayor parte de la minoría rusa lo hizo en los 90, debido a la crisis económica.

El distrito de Kentron es el centro de Ereván, y ha sufrido transformaciones importantes desde comienzos de siglo, con importantes obras de construcción y mejoras y la apertura de nuevas avenidas, como Monte Melkonian y Northern Avenue, significando la destrucción de muchos edificios históricos.

La Plaza de la República, la Ópera y la Cascada figuran entre los monumentos más importantes de la ciudad y fueron desarrollados en parte siguiendo el plan original de Tamanian y el plan de Jim Torosyan. La Plaza de la República, llamada Plaza de Lenin bajo el régimen soviético, está rodeada de cinco edificios neoclásicos en piedra toba volcánica (tuff) amarilla y rosa con motivos armenios. La Cascada, una gigantesca escalera que sale del distrito de Kentron, fue diseñada por los arquitectos Jim Torosyan, Aslan Mkhitaryan y Sargis Gurzadyan en 1971 y completada en 1980. A comienzos de este siglo fue completamente renovada y es una de los centros de vida nocturna de la ciudad. Desde su exterior se tienen magníficas vistas de Ereván y el Monte Ararat. En su cima se levanta el monumento construido en 1970 que conmemora la sovietización de Armenia y un poco más al noreste, y ubicada en una colina, en el Parque de la Victoria, la estatua de la Madre Armenia reemplaza a una estatua de Stalin erigida en 1950. Centro de las peregrinaciones para honrar los mártires armenios caídos en la 
Segunda Guerra, tiene en su base un museo militar, que en la actualidad está casi dedicado en exclusividad a la Guerra de Nagorno-Karabagh.

Las obras del Teatro de la Ópera, según un diseño de Tamanian, comenzaron en 1930 como parte de las celebraciones del 10 aniversario de la Armenia soviética, y concluyeron en 1933 aunque la sala se terminó en 1939 y el conjunto en 1953. La Ópera y la Plaza de la Libertad, llamada Plaza de la Ópera hasta 1991, que está situada al sur de la misma están ubicadas en el Parque de la Opera. La Plaza fue el escenario de manifestaciones multitudinarias a comienzos de 1988 que marcaron el comienzo del reclamo armenio para la reunificación de Nagorno-Karabagh con Armenia y también lo fue de protestas populares luego de las elecciones presidenciales en 1996, 2003, 2008 y 2013.

La más grande iglesia armenia del mundo, la Catedral de San Gregorio el Iluminador, fue consagrada en Ereván en 2001, al conmemorarse 1.700 años de cristianismo en Armenia. Las ruinas de la iglesia de Madre de Dios Katoghike del siglo VI y de la capilla de la Madre de Dios del siglo IV se encuentran en Avan, distrito al norte de Ereván, y son las más antiguas de la ciudad. Muchas de las iglesias antiguas y medievales de la ciudad fueron destruidas en los años 30 por los soviéticos. De las iglesias del centro, se destacan la iglesia Katoghike, capilla medieval de 1264 bien preservada, la iglesia de Soravor Surp Astvatsatsin construida después del terremoto de 1679 que destruyó gran parte de Ereván y la catedral de San Sarkis de 1842.

De las ocho mezquitas que había en Ereván en el siglo XIX, sólo sobrevive la Mezquita Azul, reabierta en los años 90 con apoyo de Irán y que sigue el Islam chií.

Entre los principales museos de la ciudad, fundados poco después de la sovietización de Armenia, se destacan el Matenadaran, biblioteca, museo y centro de investigación que guarda cerca de 17.000 antiguos manuscritos y biblias de la Edad Media, con una valiosa colección de antiguos manuscritos en armenio antiguo, griego antiguo, arameo, asirio, hebreo, latín y farsi; el Museo Sergei Parajanov dedicado a este cineasta soviético perseguido durante el régimen soviético; y la Galería Nacional y el Museo de Historia de Armenia en la Plaza de la República. En el primero de ellos pude visitar una exposición dedicada al pintor ruso de origen armenio Iván Aivazovsky famoso por sus paisajes marinos.

El Museo del Genocidio está ubicado en la colina de Tsitsernakaberd, en las afueras de Ereván. El memorial fue construido en 1967 y el museo en 1995. El memorial consta una estela de 44 metros que representa el renacimiento del pueblo armenio y doce losas de basalto gris que representan las provincias armenias perdidas durante el genocidio en la actual Turquía, con una llama eterna en el centro que recuerda los 1.5 millones de armenios masacrados durante el genocidio de 1915-23. En el parque, una muralla de 100 metros de largo recuerda los poblados donde ocurrieron las masacres. El 24 de abril de 1965 , en el $50^{\circ}$ aniversario del genocidio armenio, fuertes demostraciones en demanda de la recuperación las tierras armenias, tanto en Turquía como Azerbaiyán, ocurrieron en Ereván (Mutafian, 1994:145-6).

Al oeste de Ereván, y a una distancia de $18 \mathrm{~km}$, se levanta la ciudad de Vagharshapat, que es la sede Echmiadzin, la residencia del Catholicos de todos los armenios. Vagharshapat, una de las doce capitales históricas de Armenia y sede el Catholicos nuevamente desde 1441 alberga no sólo la catedral, sede del Catholicos originalmente construida en el siglo IV, sino también, entre muchos otros edificios importantes, las iglesias de San Hripsime, del siglo VII y de San Gayane también del siglo VII, joyas de la arquitectura eclesiástica armenia.

Independientemente de todos los avances a partir de 1991, Armenia tiene grandes desafíos por delante: la necesidad de una mejor coordinación con la diáspora, reducir la emigración, una mayor diversificación de la economía y de sus mercados externos y mantener a Nagorno-Karabagh en manos armenias. A pesar de todos esos desafíos, Ereván es una excelente introducción al país y muestra todas las características de una moderna capital europea. 


\section{Bibliografía}

BOURNOUTIAN, G. (2004), Eastern Armenia from the Seventeenth Century to the Russian Annexation, en HOVANNISIAN, R., Armenian People from Ancient to Modern Times, Vol. II, Foreign Dominion to Statehood, The Fifteenth Century to the Twentieth Century, St. Martin's Press, New York

CHALIAND, G. (1994), Preface, en CHORBAJIAN, L., DONABEDIAN, P., MUTAFIAN, C., The Caucasian Knot, The History and Geopolitics of Nagorno-Karabagh, Zed Books, London

CHORBAJIAN, L. (1994), Introduction to the English Language Edition, en CHORBAJIAN, L., DONABEDIAN, P., MUTAFIAN, C., The Caucasian Knot, The History and Geopolitics of NagornoKarabagh, Zed Books, London

CROISSANT, M. P. (1998), The Armenia-Azerbaijan Conflict, Causes and Implications, Praeger, Westport, CT

DASNABEDIAN, H. (2013), Historia de la Federación Revolucionaria Armenia (1890-1924), Editorial Diario Armenia, Buenos Aires

HAKOBYAN, T. (2010), Karabagh Diary, Green and Black, Neither War nor Peace, Gadarigian, Antelias, Lebanon

HOVANNISIAN, R. (2004), The Armenian Question in the Ottoman Empire 1876-1914, en HOVANNISIAN, R., Armenian People from Ancient to Modern Times, Vol. II, Foreign Dominion to Statehood, The Fifteenth Century to the Twentieth Century, St. Martin's Press, New York

HOVANNISIAN, R. (2004), Armenia's Road to Independence, en HOVANNISIAN, R., Armenian People from Ancient to Modern Times, Vol. II, Foreign Dominion to Statehood, The Fifteenth Century to the Twentieth Century, St. Martin's Press, New York

HOVANNISIAN, R. (2004), The Republic of Armenia, en HOVANNISIAN, R., Armenian People from Ancient to Modern Times, Vol. II, Foreign Dominion to Statehood, The Fifteenth Century to the Twentieth Century, St. Martin's Press, New York

KOUYMJIAN, D. (2004), Armenia from the Fall of the Cicilian Kingdom (1375) to the Forced Emigration under Shah Abbas (1604), en HOVANNISIAN, R., Armenian People from Ancient to Modern Times, Vol. II, Foreign Dominion to Statehood, The Fifteenth Century to the Twentieth Century, St. Martin's Press, New York

LIBARIDIAN, G. (1999), The Challenge of Statehood, Armenian Political Thinking since Independence, Blue Crane Books, Watertown, MA

MUTAFIAN, C. (1994), Karabagh in the Twentieth Century, en CHORBAJIAN, L., DONABEDIAN, P., MUTAFIAN, C., The Caucasian Knot, The History and Geopolitics of Nagorno-Karabagh, Zed Books, London

NALBANDIAN, L. (1963), The Armenian Revolutionary Movement, University of California Press, Berkeley

OSHAGAN, V. (2004), Modern Armenian Literature and Intellectual History from 1700 to 1915, en HOVANNISIAN, R., Armenian People from Ancient to Modern Times, Vol. II, Foreign Dominion to Statehood, The Fifteenth Century to the Twentieth Century, St. Martin's Press, New York

PANOSSIAN, R. (2006), The Armenians, From Kings and Priests to Merchants and Commissars, C. Hurst, London

RUSSELL, J. (2004), The Formation of the Armenian Nation, en HOVANNISIAN, R., Armenian People from Ancient to Modern Times, Vol. II, Foreign Dominion to Statehood, The Fifteenth Century to the Twentieth Century, St. Martin's Press, New York

SUNY, R. G. (2004), Eastern Armenians under Tsarist Rule, en HOVANNISIAN, R., Armenian People from Ancient to Modern Times, Vol. II, Foreign Dominion to Statehood, The Fifteenth Century to the Twentieth Century, St. Martin's Press, New York.

SUNY, R. G. (2004), Soviet Armenia, en HOVANNISIAN, R., Armenian People from Ancient to Modern Times, Vol. II, Foreign Dominion to Statehood, The Fifteenth Century to the Twentieth Century, St. Martin's Press, New York 
Cuadernos de Política Exterior Argentina (Nueva Época), 126, julio-diciembre 2017, pp. 79-86 ISSN 0326-7806 (edición impresa) - ISSN 1852-7213 (edición en línea)

WALKER, C. J. (2004), World War One and the Armenian Genocide, en HOVANNISIAN, R., Armenian People from Ancient to Modern Times, Vol. II, Foreign Dominion to Statehood, The Fifteenth Century to the Twentieth Century, St. Martin's Press, New York 